\title{
Antibiotic-associated Hemorrhagic Colitis Caused by Second-line Therapy for Helicobacter pylori Eradication
}

\author{
Yusaku Kajihara* \\ Department of Gastroenterology, Fuyoukai Murakami Hospital, Aomori, Japan
}

A 55-year-old man presented to the author's department with a 1-day history of abdominal pain and bloody diarrhea. Four months previously, chronic gastritis due to Helicobacter pylori was diagnosed at a medical check-up. After failure of eradication by rabeprazole-amoxicillin-clarithromycin triple therapy (first-line therapy), he was started on rabeprazole-amoxicillin-metronidazole triple therapy (secondline therapy) 5 days earlier. Vital signs were normal, and his abdomen was soft. Although laboratory evaluation showed a normal leukocyte count and a C-reactive protein of $0.5 \mathrm{mg} / \mathrm{dL}$ (range $<0.3$ ), computed tomography revealed thickening of the intestinal wall from the transverse colon to splenic flexure (Fig. 1). Colonoscopy confirmed hemorrhagic and edematous mucosa (Fig. 2). A histological evaluation of specimens obtained by biopsy showed no specific findings. Antibiotic-associated hemorrhagic colitis (AAHC) was diagnosed by a positive stool culture for Klebsiella oxytoca as well as the abovementioned findings; thus, eradication therapy was discontinued. His symptoms resolved within 2 days.

AAHC is a rare but important complication of antibiotic therapy. The etiology remains unclear; however, several mechanisms, including allergic reaction, mucosal ischemia, and microbial substitution by antibiotic-induced overgrowth of $K$. oxytoca, have been proposed. ${ }^{1}$ Symptoms include diarrhea, abdominal tenderness, and ultimately bloody diarrhea occurring within 1 week of antibiotic use; furthermore, symptoms typically disappear within 3 days after cessation of antibiotic therapy. ${ }^{2}$ Since AAHC is mainly present in the right or transverse colon, sigmoidoscopy alone would miss many cases. ${ }^{1}$

$H$. pylori infection is associated with peptic ulcers and gastric cancer. The International Agency for Research on Cancer recommends population screening for $H$. pylori and its eradication to reduce the incidence of gastric cancer. ${ }^{3}$ Additionally, the incidence rate of AAHC induced by $H$. $p y$ lori eradication therapy was reported to be $<1 \%$ [first-line therapy $0.35-0.6 \%$ vs. second-line therapy $0.96 \%$ ] in Japan. ${ }^{4}$

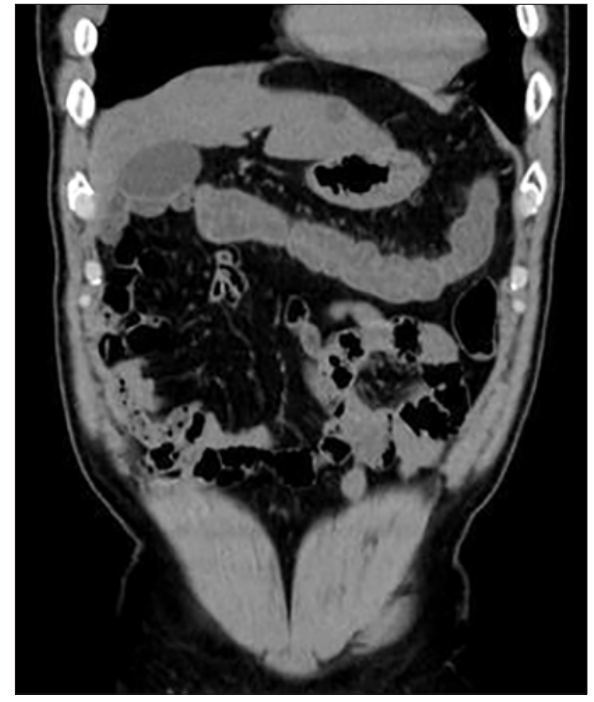

FiG. 1. Computed tomography of the transverse colon showing thickening of the intestinal wall.

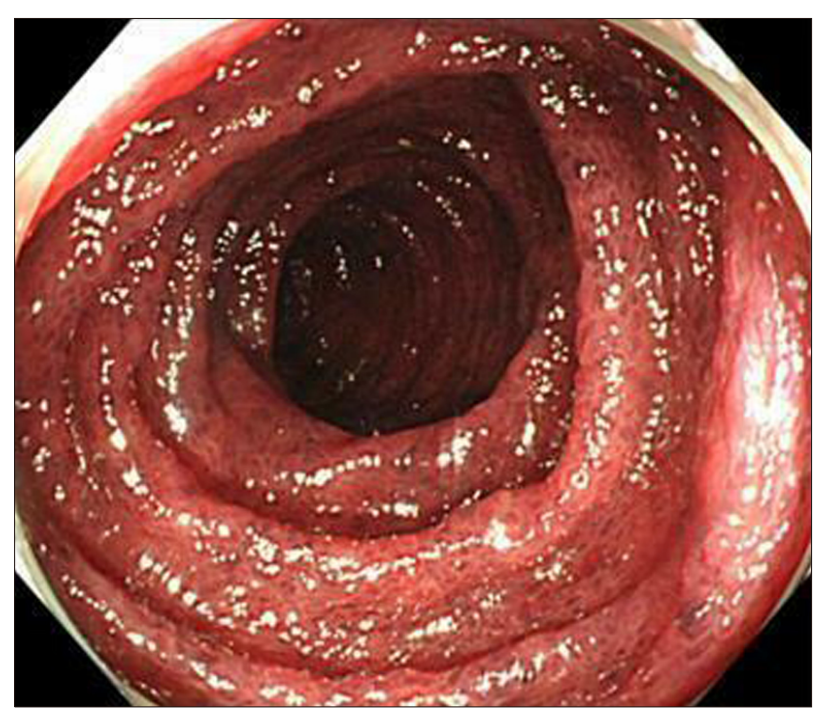

FiG. 2. Colonoscopy revealing hemorrhagic and edematous mucosa.

\section{Corresponding Author:}

Yusaku Kajihara

Department of Gastroenterology, Fuyoukai Murakami Hospital, 3-3-14 Hamada, Aomori 030-0843, Japan

Tel: +81-17-729-8888, Fax: +81-17-729-8887, E-mail: yukajihara-gi@umin.ac.jp
Article History:

Received January 9, 2020

Revised January 22, 2020

Accepted January 23, 2020 
In the present case, the important clinical messages are as follows:

(1) Physicians should be cautious of AAHC in patients taking antibiotics.

(2) Despite no adverse events associated with first-line therapy for $H$. pylori eradication, second-line therapy can cause AAHC.

(3) Colonoscopy is required for the diagnosis of AAHC.

\section{CONFLICT OF INTEREST STATEMENT}

None declared.

\section{REFERENCES}

1. Högenauer C, Langner C, Beubler E, Lippe IT, Schicho R, Gorkiewicz G, et al. Klebsiella oxytoca as a causative organism of antibioticassociated hemorrhagic colitis. N Engl J Med 2006;355:2418-26.

2. Moulis H, Vender RJ. Antibiotic-associated hemorrhagic colitis. J Clin Gastroenterol 1994;18:227-31.

3. Herrero R, Park JY, Forman D. The fight against gastric cancer the IARC Working Group report. Best Pract Res Clin Gastroenterol 2014;28:1107-14.

4. Hamada Y, Tanaka K, Yamada R, Takei Y. Hemorrhagic colitis induced by second-line Helicobacter pylori eradication. Intern Med 2020;59:301-2. 